\title{
Cronin Effect in Hadron Production off Nuclei
}

\author{
B.Z. Kopeliovich ${ }^{1,2,3}$, J. Nemchik ${ }^{4}$, A. Schäfer ${ }^{2}$ and A.V. Tarasov ${ }^{1,2,3}$ \\ 1 Max-Planck Institut für Kernphysik, Postfach 103980, 69029 Heidelberg, Germany \\ 2 Institut für Theoretische Physik der Universität, 93040 Regensburg, Germany \\ 3 Joint Institute for Nuclear Research, Dubna, 141980 Moscow Region, Russia \\ 4 Institute of Experimental Physics SAV, Watsonova 47, 04353 Kosice, Slovakia
}

(Dated: May 29, 2018)

\begin{abstract}
Recent data from RHIC for high- $p_{T}$ hadrons in gold-gold collisions raised again the long standing problem of quantitatively understanding the Cronin effect, i.e. nuclear enhancement of high- $p_{T}$ hadrons due to multiple interactions in nuclear matter. In nucleus-nucleus collisions this effect has to be reliably calculated as baseline for a signal of new physics in high- $p_{T}$ hadron production. The only possibility to test models is to compare with available data for $p A$ collisions, however, all existing models for the Cronin effect rely on a fit to the data to be explained. We develop a phenomenological description based on the light-cone QCD-dipole approach which allows to explain available data without fitting to them and to provide predictions for $p A$ collisions at RHIC and LHC. We point out that the mechanism causing Cronin effect drastically changes between the energies of fixed target experiments and RHIC-LHC. High- $p_{T}$ hadrons are produced incoherently on different nucleons at low energies, whereas the production amplitudes interfere if the energy is sufficiently high.

PACS: 24.85.+p, 13.85.Ni, 25.40.Qa
\end{abstract}

It was first observed back in 1975 [1] that high- $p_{T}$ hadrons are not suppressed in proton-nucleus collisions, but produced copiously. This effect named after James Cronin demonstrates that bound nucleons cooperate producing high- $p_{T}$ particles. Indeed, it has been soon realized that multiple interactions which have a steeper than linear $A$-dependence lead to the observed enhancement. An adequate interpretation of the Cronin effect has become especially important recently in connection with data from RHIC for high- $p_{T}$ hadron production in heavy ion collisions [2, 3]. The observed suppression factor can be understood as a product of two terms. One is due to multiple interactions within the colliding nuclei, analogous to the Cronin effect. The second factor arises from final state interaction with the produced medium, the properties of which are thus probed. This second factor, the main goal of the experiment, can be extracted from data only provided that the Cronin effect for nuclear collisions can be reliably predicted. However, in spite of the qualitative understanding of the underlying dynamics of this effect, no satisfactory quantitative explanation of existing $p A$ data has been suggested so far. Available models contain parameters fitted to the data to be explained (e.g. see [4, 5, 6]) and miss important physics. In this paper we suggest a comprehensive description of the dynamics behind the Cronin effect resulting in parameterfree predictions which agree with available data.

First of all, the mechanism of multiple interactions significantly changes with energy. At low energies a high$k_{T}$ parton is produced off different nucleons incoherently, while at high energies it becomes a coherent process. This is controlled by the coherence length

$$
l_{c}=\frac{\sqrt{s}}{m_{N} k_{T}},
$$

where $k_{T}$ is the transverse momentum of the parton produced at mid rapidity and then hadronizing into the detected hadron with transverse momentum $p_{T}$.

For a coherence length which is shorter than the typical internucleon separation, the projectile interacts incoherently with individual nucleons, just as for e.g. $p p$ scattering. However, QCD factorization is violated by multiple scattering as discussed e.g. in [7]. Therefore, broadening of transverse momentum caused by initial/final interactions, should not be translated into a modification of the parton distribution of the nucleus if the coherence length is short. In the opposite limit, i.e. if the coherence length is longer than the nuclear radius $R_{A}$, factorization applies. All amplitudes interfere coherently and result in a collective parton distribution of the nucleus. This difference is present in all of the various manners in which such interactions are discussed. It is e.g. adequate to view a nucleus in the nucleus momentum frame as a cloud of partons. Those with small $x$ overlap and are no longer associated with any individual nucleon. Small $x$ corresponds to a long $l_{c} \sim 1 /\left(x m_{N}\right)$. Again, factorization applies, but the nuclear parton distribution is modified. The mean transverse momentum of gluons increase [8] since their density saturates at small $k_{T}[9,10]$.

Short coherence length. Broadening of transverse momentum of a projectile parton propagating through a nuclear medium is quite a complicated process involving rescatterings of the parton accompanied by gluon radiation. Apparently, this process involves soft interactions and cannot be calculated perturbatively. Instead, one should rely on phenomenology. Corresponding calculations have been performed in [11] in the framework of the light-cone QCD dipole approach. The transverse momentum distribution of partons after propagation through 
nuclear matter of thickness $T_{A}(b)=\int_{-\infty}^{\infty} d z \rho_{A}(z)$ (the nuclear density integrated along the parton trajectory at impact parameter $b$ ) has the form [11,

$$
\begin{aligned}
\frac{d N_{q}}{d^{2} k_{T}} & =\int d^{2} r_{1} d^{2} r_{2} e^{i \vec{k}_{T}\left(\vec{r}_{1}-\vec{r}_{2}\right)} \Omega_{i n}^{q}\left(\vec{r}_{1}, \vec{r}_{2}\right) \\
& \times e^{-\frac{1}{2} \sigma_{\bar{q} q}^{N}\left(\vec{r}_{1}-\vec{r}_{2}, x\right) T_{A}(b)}
\end{aligned}
$$

Here $\Omega_{i n}^{q}\left(\vec{r}_{1}, \vec{r}_{2}\right)$ is the density matrix describing the impact parameter distribution of the quark in the incident hadron,

$$
\Omega_{i n}^{q}\left(\vec{r}_{1}, \vec{r}_{2}\right)=\frac{\left\langle k_{0}^{2}\right\rangle}{\pi} e^{-\frac{1}{2}\left(r_{1}^{2}+r_{2}^{2}\right)\left\langle k_{0}^{2}\right\rangle},
$$

where $\left\langle k_{0}^{2}\right\rangle$ is the mean value of the parton primordial transverse momentum squared.

The central ingredient of Eq. (2) is the phenomenological cross section $\sigma_{\bar{q} q}^{N}\left(r_{T}, x\right)$ for the interaction of a nucleon with a $\bar{q} q$ dipole of transverse separation $r_{T}$ at Bjorken $x$. In what follows we use the simple parametrization 12

$$
\sigma_{\bar{q} q}\left(r_{T}, x\right)=\sigma_{0}\left[1-e^{-\frac{1}{4} r_{T}^{2} Q_{s}^{2}(x)}\right],
$$

the parameters of which were fixed by DIS data: $Q_{s}(x)=$ $1 \mathrm{GeV} \times\left(x_{0} / x\right)^{\lambda / 2}$ and $\sigma_{0}=23.03 \mathrm{mb} ; \lambda=0.288 ; x_{0}=$ $3.04 \cdot 10^{-4}$.

Note that the $k_{T}$ distribution of quarks from a single $q-N$ scattering process is not singular at $k_{T} \rightarrow 0$, but according to (1) has a Gaussian shape. The phenomenon of saturation for soft gluons [9, 13 is the driving idea of parametrization [12]. Therefore, the mean momentum transfer in each scattering is not small, but of the order of the saturation scale $Q_{s}(x)$.

Of course, for projectile gluons the broadening is stronger than for quarks and the dipole cross section Eq. (11) should be replaced by the glue-glue one $\sigma_{G G}^{N}=$ $\frac{9}{4} \sigma_{\bar{q} q}^{N}$.

Besides broadening of transverse momentum, initial state interactions also lead to energy loss [14, 15]. While induced energy loss in cold nuclear medium is negligibly small [15, 16], energy loss due to hadronization in inelastic scattering reactions (which is basically the same as for hadronization in vacuum) is important. The first inelastic interaction of the incident hadron triggers energy loss and the parton participating in the high- $p_{T}$ process arrives with a noticeably reduced energy 14, 15. We fixed the energy loss $\Delta E$ to a mean value corresponding to the mean path length calculated in [15] and a rate of energy loss $d E / d z=-2.5 \mathrm{GeV} / \mathrm{fm}$.

For the cross section of $p A \rightarrow h X$ at high $p_{T}$ we use the standard convolution expression based on QCD factorization [17,

$$
\sigma_{p A}^{l_{c} \ll R_{A}}\left(p_{T}\right)=\sum_{i, j, k, l} \widetilde{F}_{i / p} \otimes F_{j / A} \otimes \hat{\sigma}_{i j \rightarrow k l} \otimes D_{h / k},
$$

where $F_{i / p}$ and $F_{j / A}$ are the distributions of parton species $i, j$ in Bjorken $x_{1,2}$ and transverse momentum in the colliding proton and nucleus respectively. However, to describe the nonfactorizable multiple interactions the beam parton distribution $\widetilde{F}_{i}^{p}$ is modified by by the transverse momentum broadening Eq. (2) and by shifting $x_{1}$ to $\tilde{x}_{1}=x_{1}+\Delta E /\left(x_{1} E_{p}\right)$. For $\left\langle k_{0}^{2}\right\rangle$ in (3) we use the next-to-leading value from [5] fitted to data for hadron production in $p p$ collisions. For the parton distribution functions in a nucleon we use the leading order GRV parametrization [18. The nuclear parton distribution, $F_{j / A}$, is unchanged compared to a free nucleon, except at large $x_{2}$ where it is subject to medium modifications (EMC effect) which are parametrized according to [19. For the hard parton scattering cross section [17] we use regularization masses $m_{G}=0.8 \mathrm{GeV}$ and $m_{q}=0.2 \mathrm{GeV}$ for gluon and quark propagators respectively. Such a large effective gluon mass was introduced to reproduce the strong nonperturbative light-cone gluon interaction [20] dictated by diffraction data. The fragmentation functions of a parton $k$ into the final hadron $h, D_{h / k}$ are taken from 21] in leading order. We use the realistic Woods-Saxon parametrization for the nuclear density.

As far as all the parameters in (5) are fitted to data for proton target, we have no further adjustable parameters and can predict nuclear effect. The results of parameterfree calculations for the production of charged pions are compared in Fig. 1 1 with fixed target data. $R_{W / B e}\left(p_{T}\right)$ is the ratio of the tungsten and beryllium cross sections at $200-400 \mathrm{GeV}$ [22] and $800 \mathrm{GeV}$ [23] as function of $p_{T}$.

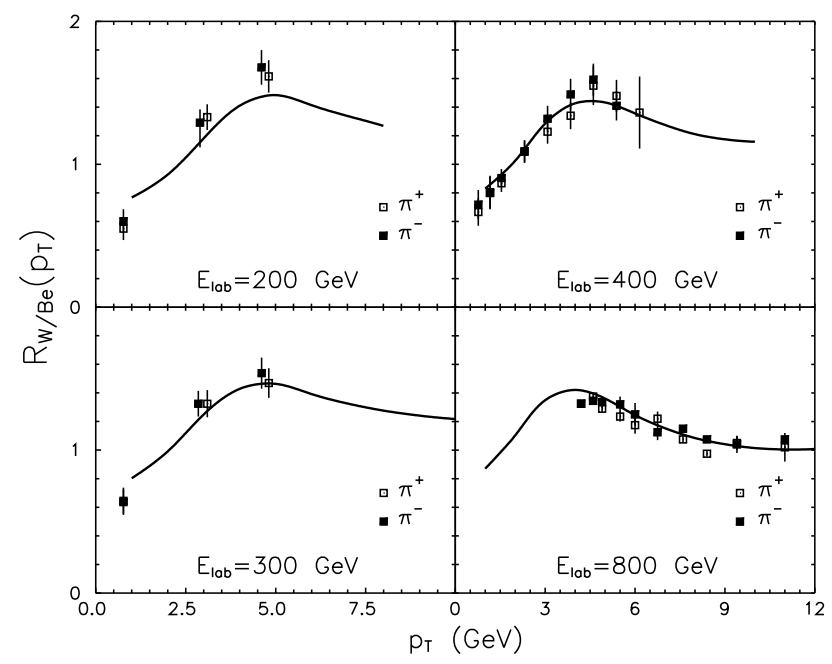

FIG. 1: Ratio of the charged pion production cross sections for tungsten and beryllium function of the transverse momentum of the produced pions. The curves correspond to the parameter-free calculation Eq. (5), the data are from fixed target experiments 22, 23.

Long coherence length. In the limit of $l_{c} \gg R_{A}$ a hard fluctuation in the incident proton containing a high- $p_{T}$ parton propagates through the whole nucleus and may 
be freed by the interaction. Since multiple interactions in the nucleus supply a larger momentum transfer than a nucleon target, they are able to resolve harder fluctuations, i.e. the average transverse momentum of produced hadrons increases. In this case broadening looks like color filtering rather than Brownian motion.

Instead of QCD factorization we employ the light-cone dipole formalism in the rest frame of the target which leads to another factorized expression, valid at $x_{2} \ll 1$,

$$
\sigma_{p A}^{l_{c} \gg R_{A}}\left(p_{T}\right)=F_{G / p} \otimes \sigma\left(G A \rightarrow G_{1} G_{2} X\right) \otimes D_{h / G_{1}} .
$$

We assume that high- $p_{T}$ hadrons originate mainly from radiated gluons at such high energies. The cross section of gluon radiation reads [20, 25, 26],

$$
\begin{aligned}
& \frac{d \sigma\left(G A \rightarrow G_{1} G_{2} X\right)}{d^{2} p_{T} d y_{1}}=\int d^{2} b \int d^{2} r_{1} d^{2} r_{2} e^{i \vec{p}_{T}\left(\vec{r}_{1}-\vec{r}_{2}\right)} \\
\times & \frac{\Psi_{G G}^{*}\left(\vec{r}_{1}, \alpha\right) \Psi_{G G}\left(\vec{r}_{2}, \alpha\right)}{1}\left[1-e^{-\frac{1}{2} \sigma_{3 G}^{N}\left(r_{1}, x\right) T_{A}(b)}\right. \\
- & \left.e^{-\frac{1}{2} \sigma_{3 G}^{N}\left(r_{2}, x\right) T_{A}(b)}+e^{-\frac{1}{2} \sigma_{3 G}^{N}\left(\vec{r}_{1}-\vec{r}_{2}, x\right) T_{A}(b)}\right] .
\end{aligned}
$$

Here $\alpha=p_{+}\left(G_{1}\right) / p_{+}(G)$ is the momentum fraction of the radiated gluon; $\sigma_{3 G}^{N}(r, \alpha)$ is the dipole cross section for a three-gluon colorless system, where $\vec{r}$ is the transverse separation of the final gluons $G_{1}$ and $G_{2}$. It can be expressed in terms of the usual $\bar{q} q$ dipole cross sections,

$$
\sigma_{3 G}^{N}(r)=\frac{2}{9}\left\{\sigma_{\bar{q} q}(r)+\sigma_{\bar{q} q}(\alpha r)+\sigma_{\bar{q} q}[(1-\alpha) r]\right\} .
$$

The light-cone wave function of the $G_{1}-G_{2}$ Fock component of the incoming gluon including the nonperturbative interaction of the gluons reads [20],

$$
\begin{array}{r}
\Psi_{G G}(\vec{r}, \alpha)=\frac{\sqrt{8 \alpha_{s}}}{\pi r^{2}} \exp \left[-\frac{r^{2}}{2 r_{0}^{2}}\right]\left[\alpha\left(\vec{e}_{1}^{*} \cdot \vec{e}\right)\left(\vec{e}_{2}^{*} \cdot \vec{r}\right)\right. \\
\left.+(1-\alpha)\left(\vec{e}_{2}^{*} \cdot \vec{e}\right)\left(\vec{e}_{1}^{*} \cdot \vec{r}\right)-\alpha(1-\alpha)\left(\vec{e}_{1}^{*} \cdot \vec{e}_{2}^{*}\right)(\vec{e} \cdot \vec{r})\right],
\end{array}
$$

where $r_{0}=0.3 \mathrm{fm}$ is the parameter characterizing the strength of the nonperturbative interaction which was fitted to data on diffractive $p p$ scattering. The product of the wave functions is averaged in (7) over the initial gluon polarization, $\vec{e}$, and summed over the final ones, $\vec{e}_{1,2}$.

Expression (7) with the exponentials expanded to first order in the nuclear thickness also provides the cross section for gluon radiation in $p p$ collisions. This cross section reproduces well the measured pion spectra in $p p$ collisions. The results for the ratio of pion production rates in $p A$ and $p p$ collisions obtained using Eqs. (6)-(7) for mid rapidity at the energy of LHC, $\sqrt{s}=5.5 \mathrm{TeV}$ are shown by curve in Fig. 2 .

Note that at the high LHC energy the eikonal formula Eq. (7) is not exact. The higher Fock components

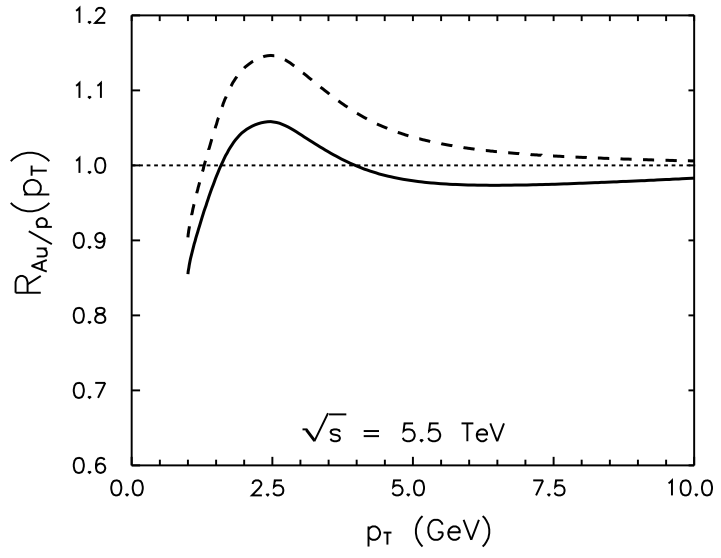

FIG. 2: Ratio of $p-A u$ to $p p$ cross sections as function of transverse momentum of produced pions at the energy of LHC calculated with Eq. (7). The dashed and solid curves correspond to calculations without and with gluon shadowing respectively.

$|3 G\rangle,|4 G\rangle$, etc. lead to additional corrections called gluon shadowing. These fluctuations are heavier than $|2 G\rangle$, correspondingly, the coherence length is shorter, and one should sum over all different trajectories of the gluons. This problem was solved in 20, 24, 27] and a suppression factor $R_{G}\left(x, Q^{2}, b\right)$ due to gluon shadowing was derived. Here we make use of those results replacing the dipole cross sections in (7), $\sigma_{3 G}$ by $R_{G} \sigma_{3 G}$. This suppression factor leads to a reduction of the Cronin effect as is demonstrated by the solid curve in Fig. 2. Note that this curve approaches unity from below at high $p_{T}$.

Predictions for RHIC. The calculations in the energy range of RHIC are most complicated since this is the transition region between the regimes of long $\left(\operatorname{small} p_{T}\right)$ and short (large $p_{T}$ ) coherence lengths. One can deal with this situation relying on the light-cone Green function formalism [27, 28, 29]. However, in this case the integrations involved become too complicated. Fortunately, the coherence length at the energy of RHIC is rather long, $l_{c} \sim 5 \mathrm{fm}$, within the $p_{T}$-range where the Cronin effect has an appreciable magnitude. Therefore, the corrections to the asymptotic expression Eq. (6) should not be large and can be approximated by linear interpolation performed by means of the the so called nuclear longitudinal formfactor $F_{A}\left(q_{c}, b\right)$ [15, 30],

$$
\begin{aligned}
\sigma_{p A}\left(p_{T}\right) & =\int d^{2} b\left\{\left[1-\left\langle F_{A}^{2}\left(q_{c}, b\right)\right\rangle\right] \sigma_{p A}^{l_{c} \ll R_{A}}\left(p_{T}, b\right)\right. \\
& \left.+\left\langle F_{A}^{2}\left(q_{c}, b\right)\right\rangle \sigma_{p A}^{l_{c} \gg R_{A}}\left(p_{T}, b\right)\right\} .
\end{aligned}
$$

Here $\sigma_{p A}\left(p_{T}, b\right)$ is the unintegrated $\vec{b}$-dependent contribution to the cross section $\sigma_{p A}\left(p_{T}\right)$,

$$
F_{A}\left(q_{c}, b\right)=\frac{1}{T_{A}(b)} \int_{-\infty}^{\infty} d z \rho_{A}(b, z) e^{i q_{c} z}
$$


where $q_{c}=1 / l_{c}$. The formfactor is averaged weighted with the cross section at fixed $p_{T}$ and varying initial and final parton momenta.

Expression (10) interpolates between the cross sections $\sigma_{p A}^{l_{c} \ll R_{A}}\left(p_{T}\right)$, Eq. (5), and $\sigma_{p A}^{l_{c} \gg R_{A}}\left(p_{T}\right)$, Eq. (6), which are shown in Fig. 3 by dotted and dashed curves respectively. It is interesting that the dashed curve exposes a weaker

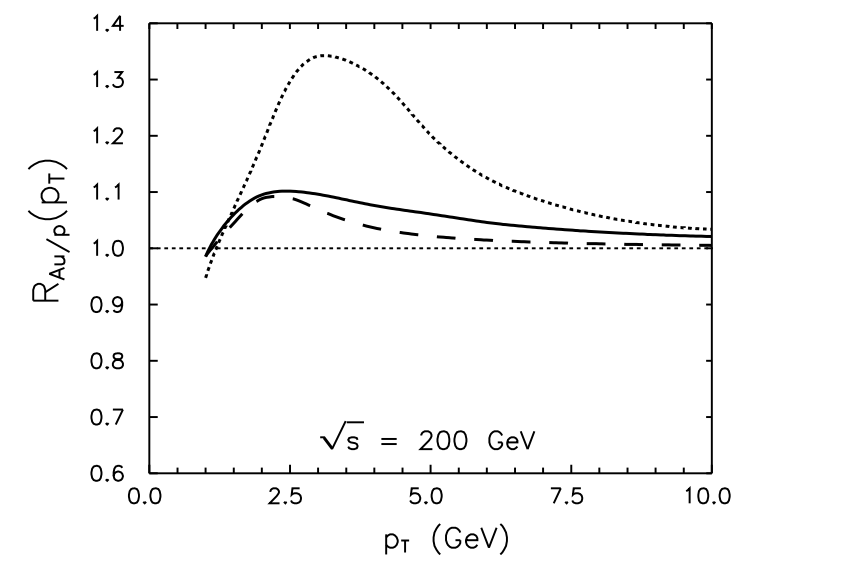

FIG. 3: Predictions for RHIC. The dotted and dashed curves are calculated at $\sqrt{s}=200 \mathrm{GeV}$ using Eqs. (月) and (6) respectively. The final prediction taking into account the coherence length is shown by the solid curve.

nuclear enhancement than the dotted one. This might be interpreted as Landau-Pomeranchuk suppression of the radiation spectrum compared to the Bethe-Heitler regime.

Our prediction for $\sqrt{s}=200 \mathrm{GeV}$ calculated with Eq. (10) is depicted by the solid curve which nearly coincides with the $l_{c} \ll R_{A}$ one at $p_{T}<2 \mathrm{GeV}$ and is rather close to it at higher $p_{T} . \quad l_{c} \gg R_{A}$ regime at higher $p_{T}$. Eventually, all three curves approach 1 at large $p_{T}>10 \mathrm{GeV}$.

No sizeable gluon shadowing is expected at RHIC energy. The reason is that the effective coherence length for gluon shadowing evaluated in 29] is nearly an order of magnitude shorter than $l_{c}$ for single gluon radiation as given by (1).

Summary: the mechanism of high- $p_{T}$ hadron production has two limiting regimes. At $l_{c} \ll R_{A}$ a high- $p_{T}$ particle is produced incoherently on different nucleons, and the Cronin effect is due to soft multiple initial/final state interactions which break QCD factorization. On the contrary, for $l_{c} \gg R_{A}$ the process of gluon radiation takes long time even for high transverse momenta. As a result, coherent radiation from different nucleons is subject to Landau-Pomeranchuk suppression. Using the light-cone dipole approach we provided the first parameter-free calculations for the Cronin effect in $p A$ collisions, i.e. no fit is done to the data to be described. Our results agree well with available data and we provided predictions for high- $p_{T}$ pion production at RHIC and LHC.
Acknowledgment: we are grateful to Jörg Hüfner, Mikkel Johnson and Jörg Raufeisen for stimulating discussions. This work has been partially supported by a grant from the Gesellschaft für Schwerionenforschung Darmstadt (GSI), grant No. GSI-OR-SCH. The work of J.N. has been supported in part by the Slovak Funding Agency, Grant No. 2/1169 and Grant No. 6114.

[1] J. W. Cronin et al., Phys. Rev. D 11, 3105 (1975).

[2] PHENIX Collaboration, K. Adcox, et al., nuclex/0109003 (2001).

[3] STAR Collaboration, J. Harris et al., nucl-ex/0105011 (2001).

[4] T. Ochiai et al., Prog. Theor. Phys., 75, 288 (1986).

[5] X.-N. Wang, Phys. Rev. C 61, 064910 (2000).

[6] Y. Zhang et al., Phys.Rev. C 65, 034903 (2002).

[7] G.T. Bodwin, S.J. Brodsky, and G.P. Lepage, Phys. Rev. D 39, 3287 (1989).

[8] L. McLerran and R. Venugopalan, Phys. Rev. D 49, 2233 (1994); 49, 3352 (1994); 49, 2225 (1994).

[9] L.V. Gribov, E.M. Levin and M.G. Ryskin, Phys. Rep. 100, 1 (1983).

[10] A.H. Mueller, Nucl. Phys. B 335, 115 (1990).

[11] M.B. Johnson, B.Z. Kopeliovich, and A.V. Tarasov, Phys. Rev. C63, 035203 (2001).

[12] K. Golec-Biernat and M. Wüsthoff, Phys. Rev. D 59, 014017 (1999); Phys. Rev. D 60, 114023 (1999).

[13] A.H. Mueller, Nucl. Phys. B 558, 285 (1999).

[14] B.Z. Kopeliovich, F. Niedermayer, Sov. J. Nucl. Phys. 42, 504 (1985) [Yad. Fiz. 42, 797 (1985)].

[15] M.B. Johnson et al., Phys.Rev.Lett. 86, 4483 (2001); Phys. Rev. C 65, 025203 (2002).

[16] R. Baier et al., Nucl. Phys. B 483, 291 (1997).

[17] R.P. Feynman, R.D. Field, and G.C. Fox, Phys. Rev. D 18, 3320 (1978).

[18] M. Gluck, E. Reya, and A. Vogt, Z. Phys. C 67, 433 (1995).

[19] K. J. Eskola, V. J. Kolhinen, and C.A. Salgado, Eur. Phys. J. C 9, 61 (1999).

[20] B.Z. Kopeliovich, A. Schäfer and A.V. Tarasov, Phys. Rev. D 62, 054022 (2000).

[21] B.A. Kniehl, G. Kramer, and B. Pötter, B 597, 337 (2001).

[22] D. Antreasyan et al., Phys. Rev. D 19, 764 (1979).

[23] P.B. Straub et al., Phys. Rev. Lett. 68, 452 (1992).

[24] B.Z. Kopeliovich, J. Raufeisen, A.V. Tarasov, and M.B. Johnson, hep-ph/0110221.

[25] Yu.V. Kovchegov and A.H. Mueller, Nucl. Phys. B 529, 451 (1998).

[26] B.Z. Kopeliovich, A. Schäfer and A.V. Tarasov, Phys. Rev. C 59, 1609 (1999).

[27] B.Z. Kopeliovich, J. Nemchik, A. Schäfer and A.V. Tarasov, Phys. Rev. C 65, 035201 (2002).

[28] B.Z. Kopeliovich, J. Raufeisen, A.V. Tarasov, Phys. Lett. B 440, 151 (1998);

[29] B.Z. Kopeliovich, J. Raufeisen, A.V. Tarasov, Phys. Rev. C 62, 035204 (2000).

[30] B.Z. Kopeliovich, B. Povh, Phys. Lett. B 367, 329 (1996). 